\title{
EFEITO DO MANEJO DE PLANTAS DANINHAS SOBRE Neoseiulus californicus (ACARI: PHYTOSEIIDAE) EM POMAR DE MACIEIRA ${ }^{1}$
}

\author{
LINOBITTENCOURTMONTEIRO ${ }^{2}$, LUIZBELLI $^{3}$, ALEXANDER DE SOUZA $^{4}$, ANDRÉ LUIZ WERNER ${ }^{4}$
}

RESUMO - Avaliou-se a influência do manejo de plantas daninhas sobre o deslocamento de ácaros tetraniquídeos (Panonychus ulmi e Tetranychus urticae) e do ácaro predador Neoseiulus californicus em um pomar de macieira 'Gala', onde foi implantado o controle biológico do ácaro vermelho, $P$. ulmi, por meio de liberações massais de $N$. californicus. As parcelas tiveram as plantas daninhas manejadas de três formas: sem manejo, com roçadas manuais e com herbicidas. As populações de ácaros foram avaliadas sobre as plantas daninhas, Plantago tormentosa e Erigeron sp, e sobre as folhas de macieira. As maiores populações de $N$. californicus foram observadas nas parcelas onde os manejos proporcionaram desenvolvimento de plantas daninhas na linha de plantio. Na parcela manejada com herbicida, houve maior população de ácaros tetraniquídeos sobre as macieiras, provavelmente, devido ao reduzido número de $N$. californicus. $P$. tormentosa foi o hospedeiro preferencial do ácaro predador. Concluiu-se que o manejo de plantas daninhas, na linha de plantio das macieiras, assume um importante papel no equilíbrio entre as populações de ácaros.

Termos para indexação: controle biológico, manejo plantas daninhas, fitoseídeos, macieira

\section{EFFECT OF WEED MANAGEMENT ON Neoseiulus californicus (ACARI: PHYTOSEIIDAE) IN APPLE ORCHARD}

ABSTRACT - The influence of weed management on tetranychid mites (Panonychus ulmi and Tetranychus urticae) and phytoseids movement was evaluated. Neoseiulus californicus populations were released in an apple orchard for biological control of tetranychid mites. Three kinds of weed management were used: no weed control at all, manual control and control with herbicide. The mites were evaluated in Plantago tormentosa, Erigeron $s p$, and apple tree leaves. The highest population of $N$. californicus was observed where weed was not controlled. Tetranychid mites populations were abundant on apple trees when herbicide was used, probably due to the low population of $N$. californicus. P. tormentosa was the preferred host of phytoseids. The conclusion was that weed management plays an important role in the regulation of mite species in apple orchards.

Index terms: biological control, weed manegement, phytoseid, apple

\section{INTRODUÇÃO}

Os desequilíbrios ambientais causados pelo uso indiscriminado de agroquímicos têm acarretado um aumento da população de ácaros fitófagos nas culturas comerciais em todo o mundo (Van de Vrie, 1985). Em todas as regiões produtoras de maçãs do Brasil, 3 a 4 pulverizações de acaricidas por ciclo vegetativo têm sido realizadas, muitas vezes, de forma preventiva para o controle de ácaros fitófagos (Monteiro, 1994). Na região de Fraiburgo, em Santa Catarina, o ácaro vermelho, Panonychus ulmi (Koch) (Acari: Tetranychidae), é uma importante praga em macieira. O ácaro vermelho hiberna na forma de ovos sobre os ramos da macieira e as primeiras eclosões de larvas ocorrem em meados de setembro (Carvalho, 1971). Em novembro, a população alcança o nível de controle, provavelmente, devido ao desequilíbrio do agroecossistema (Vet \& Dicke, 1992). Uma alternativa ao controle químico preventivo é o manejo integrado de pragas com a utilização de inimigos naturais. Em 1995, o controle biológico do ácaro vermelho foi implantado em pomares de macieiras em Fraiburgo, Santa Catarina, por meio de criações do ácaro predador Neoseiulus californicus (McGregor) (Acari: Phytoseiidae) em estufas e de liberações inoculativas. O ácaro predador permanece sobre as folhas da macieira durante quase todo o ciclo vegetativo da cultura, passando o inverno sobre plantas daninhas (Fauvel et al. 1993).

Normalmente, o controle de plantas daninhas é realizado com herbicidas na linha de plantio, visando a facilitar os tratos culturais; em conseqüência, o solo fica sem vegetação desde o outono até meados da primavera. As plantas daninhas possuem um papel importante na manutenção de inimigos naturais, devido ao fornecimento de abrigo, de umidade e de alimento (Altieri \& Liebman 1986).

O objetivo deste trabalho foi determinar o impacto do manejo de plantas daninhas sobre a população de ácaros em pomares de macieira.

\section{MATERIALEMÉTODOS}

O experimento foi conduzidos na Agrícola Fraiburgo SA., Fraiburgo (SC). A variedade comercial do pomar era Gala, com a variedade Fuji como polinizadora, em uma área de plantas com 9 anos de idade. $\mathrm{O}$ espaçamento de plantio foi de $1 \mathrm{~m}$ entre plantas e de 4,5 $\mathrm{m}$ entre as linhas. Esta área estava sendo conduzida com o controle biológico de ácaros, cujo ácaro predador, $N$. californicus, foi criado massalmente em estufa, alimentando-se de Tetranychus urticae Koch (Acari: Tetranychidae). Entre novembro de 1997 e abril de 1998, foram realizadas 3 liberações de $N$. californicus na área do experimento, totalizando aproximadamente 250000 formas móveis.

O experimento teve três tratamentos, correspondendo aos manejos de plantas daninhas realizados na linha de plantio, definida como sendo a área da projeção de copa foliar, com bordadura afastada $0,80 \mathrm{~m}$ do tronco da macieira. Cada tratamento consistiu de 5 linhas de plantio de macieiras contendo 12 plantas em cada linha. As amostragens foram realizadas em uma área delimitada entre as 3 linhas centrais, deixando o restante como bordadura. As repetições foram definidas como sendo as plantas daninhas e as macieiras observadas. O delineamento experimental foi inteiramente casualisado. Os dados obtidos foram submetidos à análise de variância e as médias foram comparadas pelo teste de Duncan, ao nível de 5\% de probabilidade. Os tratamentos foram conduzidos da seguinte forma:

Parcela herbicida (PH) - o manejo de plantas daninhas, na linha de plantio, foi realizado com glifosate, na concentração de $4 \%$ de produto comercial, nos dias 17 de agosto e 23 de outubro de 1998 e 12 de janeiro de 1999, de maneira que a área permaneceu sem vegetação durante todo o ciclo.

Parcela roçada (PR) - o manejo de plantas daninhas ocorreu por meio de roçadeira manual, realizada tantas vezes quanto necessárias, a fim de manter a vegetação com $0,20 \mathrm{~m}$ de altura. $\mathrm{O}$ manejo de plantas daninhas nas entrelinhas foi realizado com uma roçadeira acoplada a um trator. A faixa de roçada foi de 2,9 $\mathrm{m}$ de largura.

Parcela sem manejo (PSM) - não houve controle de plantas daninhas. Para facilitar os tratos culturais, houve uma roçada em janeiro; entretanto, as plantas daninhas foram mantidas a uma altura maior do que $0,40 \mathrm{~m}$. Ácaros sobre plantas daninhas - As observações sobre as plantas daninhas foram realizadas em tanchagem (Plantago tormentosa) e buva (Erigeron sp), nos dias 9-11-98, 14-12-98, 15-01-99, 1-2-99, 18-2-99, 19-399 e 14-5-99. Para cada tratamento, foram coletadas 6 plantas daninhas,

1 (Trabalho 022/2001). Recebido: 16/01/2001. Aceito para publicação: 06/09/2002.

2 Prof. Adjunto do Departamento de Fitotecnia e Fitossanitarismo, UFPR, C. Postal 19061, CEP 81.531-990, Curitiba, PR - lbmonte@ terra.com.br

3 Técnico Agrícola, Agrícola Fraiburgo S.A., Rod. SC 453, Km 35, CEP 89580-000, Fraiburgo, SC

4 Eng $^{\circ}$. Agro., Agrícola Fraiburgo S. A., Rod. SC 453, Km 35, CEP 89580-000, Fraiburgo, SC 
retirando-as do solo por meio de um corte junto às raízes e contando os ácaros presentes em todas as folhas com o auxílio de um estereomicroscópio (10 X). Não foi levado em consideração o estágio de desenvolvimento da planta daninha ou o número de folhas, porque ocorreram variações de tamanho das ervas daninhas em todos os tratamentos. As plantas daninhas, nos três tratamentos, foram retiradas sobre a projeção da copa da macieira; na parcela herbicida, não havendo exemplares na linha de plantio, as plantas foram coletadas nas entrelinhas, o mais próximo possível da projeção da copa. Todas as observações foram realizadas entre as 10 e $15 \mathrm{~h}$, quando o deslocamento diurno de ácaros é considerado mínimo (Purvis \& Curry, 1981).

Ácaros nas folhas de macieira - Seis macieiras, localizadas na linha de plantio central de cada tratamento, foram utilizadas para a coleta de 30 folhas de macieira, localizadas no terço médio dos ramos do ano e situadas à altura de 1,7 m,. As folhas foram levadas ao laboratório para a contagem de todas as fases de ácaros predadores e fitófagos, com o auxílio de um estereomicroscópio (10 X), sendo os mesmos identificados e separados em dois grupos: $N$. californicus e tetraniquídeos (T. urticae e P. ulmi). As amostragens foram realizadas nos dias 19-10-98, 23-11-98, 21-12-98, 6-1-99, 20-1-99, 18-2-99 e 14-5-99. O nível de controle foi estabelecido em $50 \%$ de folhas com presença de ácaros fitófagos, não levando em consideração o número de ácaros por folha. O controle de ácaros foi realizado com Omite $720 \mathrm{CE}$ (propargite), na concentração de $100 \mathrm{ml} / \mathrm{L}$ (4/1/99), pois este acaricida está sendo utilizado em pomares de macieira desde 1992, e não tem apresentado efeitos negativos sobre o desenvolvimento de N. californicus (Monteiro, 1993; 1994).

\section{RESULTADOSE DISCUSSÃO}

Ácaros sobre plantas daninhas - Nas observações sobre as plantas daninhas, foi encontrado $71 \%$ de $T$. urticae, entre os tetraniquídeos. Estes ácaros foram mais abundantes sobre $P$. tormentosa desenvolvendo-se na parcela sem manejo (PSM) (Tabela 1), o que era esperado, por possuir o hábito de passar o inverno em substrato herbáceo, sendo que sua migração e atividade aumentam com a temperatura (Baillod \& Guignard, 1980). No final do inverno, a maior população de tetraniquídeos em PSM, está relacionada com a abundância de plantas daninhas, contrastando com a reduzida população na parcela herbicida (PH) (Tabela 1).

TABELA 1 - Flutuação de Neoseiulus californicus e tetraniquídeos sobre Plantago tormentosa e Erigeron $s p$. em três manejos de plantas daninhas em pomares de macieira, Agrícola Fraiburgo S.A., Fraiburgo, 1998.

\begin{tabular}{|c|c|c|c|c|c|c|c|c|c|c|c|c|}
\hline \multirow{3}{*}{ Data } & \multicolumn{6}{|c|}{ Neoseiulus californicus $^{l}$} & \multicolumn{6}{|c|}{ Tetraniquídeos } \\
\hline & \multicolumn{3}{|c|}{ Plantago tormentosa } & \multicolumn{3}{|c|}{ Erigeron $s p$} & \multicolumn{3}{|c|}{ Plantago tormentosa } & \multicolumn{3}{|c|}{ Erigeron $s p$} \\
\hline & PSM & PR & PH & PSM & PR & PH & PSM & PR & PH & PSM & PR & PH \\
\hline 9-nov- & $6,7 \mathrm{~B}$ & $8,2 \mathrm{~A}$ & $1,5 \mathrm{C}$ & $5,7 \mathrm{~B}$ & $1,0 \mathrm{C}$ & 1,8 & $8 \mathrm{~A}$ & $3,3 \mathrm{~B}$ & 0,3 & $3,8 \mathrm{~B}$ & $2,7 \mathrm{~B}$ & $1,5 \mathrm{C}$ \\
\hline 14-de & 0, & 0,0 & 0 & 0,0 & 0, & 0 , & & 0,0 & 0 , & 0,0 & 0,0 & 0,0 \\
\hline $5-\mathrm{j}$ & 0,0 & 0,0 & 0,0 & 0,0 & 0, & 0 , & & 0,0 & 0 , & 0 & 0,0 & 0,0 \\
\hline 1-fe & 0,0 & 0,0 & 0,0 & 0,0 & 0, & 0 & & 0,0 & 0, & 0,0 & 0, & 0,0 \\
\hline 18 -fe & 0,0 & 0,0 & 0,0 & 0,0 & $0, c$ & 0 & & 0,0 & 0, & 0,0 & 0 & 0,0 \\
\hline 19-mar-99 & 0,0 & 0,0 & 0,0 & 0,0 & 0,0 & 0,0 & 0,0 & 0,0 & 0,0 & 0,0 & 0,0 & 0,0 \\
\hline 14-mai-99 & $2,2 \mathrm{~B}$ & $4,2 \mathrm{~A}$ & $3,2 \mathrm{AB}$ & $0,2 \mathrm{C}$ & $0,3 \mathrm{C}$ & $0,5 \mathrm{C}$ & $4,0 \mathrm{~B}$ & $5,5 \mathrm{~A}$ & $4,5 \mathrm{AB}$ & $0,5 \mathrm{C}$ & $0,8 \mathrm{C}$ & $1,0 \mathrm{C}$ \\
\hline
\end{tabular}

${ }^{1}$ Número médio de ácaros por erva daninha

${ }^{2} \mathbf{P S M}$ - parcela sem manejo; PR - parcela roçada; $\mathbf{P H}$ - parcela herbicida.

${ }^{3}$ Médias seguidas pelas mesmas letras na linha e para cada espécie de ácaros não diferem estatisticamente, pelo teste de Duncan, a 5\% de probabilidade

Todos os ácaros fitoseídeos coletados foram da espécie $N$. californicus, não sendo encontradas outras espécies, provavelmente, devido às liberações inoculativas no pomar e nas áreas adjacentes. $N$. californicus é uma espécie dominante (Fauvel et al., 1993), podendo impedir o desenvolvimento de outros ácaros predadores nas mesmas áreas. O mesmo foi observado nos pomares da Pomifrai Fruticultura Ltda (Fraiburgo-SC), os quais apresentavam grandes populações de Phytoseiulus macropilis (Banks) (Acari: Phytoseiidae) em fevereiro de
1995; entretanto, após liberações de $N$. californicus, $P$. macropilis não foi mais encontrado nos anos subsequentes (Monteiro, informação pessoal).

Nas amostragens realizadas em 9-11-98 e 14-5-99, N. californicus foi encontrado em maior quantidades sobre $P$. tormentosa do que Erigeron sp (Tabela 1). É possível que P. tormentosa emita cairomônios mais atrativos para o ácaro predador, como demonstrado no trabalho de Collier (1998), comparando esta espécie com Sida rhombifolia e Taraxucum officinale. A permanência de $N$. californicus no solo até meados de dezembro pode estar associada aos cairomônios emitidos pelas plantas daninhas e à abundância de alimento disponível no solo (Juvara-Bals, 1992; Collier, 1998), resultado encontrado na PSM, onde houve correlação positiva $(\mathrm{p}<0,79)$ entre as populações de tetraniquídeos e de N. californicus (Castagnoli \& Simoni, 1991).

Verificou-se a importância das plantas daninhas sobre a presença de ácaros, pois as parcelas PSM e parcela roçada $(\mathbf{P R})$ tiveram as maiores populações de ambas as espécies de ácaros, exceção a $P$. tormentosa no tratamento PR para tetraniquídeos, em 14-5-99. Em se tratando de manejo de plantas daninhas, os resultados demonstram que a presença de $P$. tormentosa é importante em áreas de controle biológico, pois, além de abrigar $N$. californicus e tetraniquídeos, produz pólen, importante fonte de alimento para os ácaros predadores (Costa-Comelles et al. 1986, Castognoli et al. 1995). Além disso, P. tormentosa é uma planta de porte baixo que não interferiria nos tratos culturais realizados em pomares de macieira, a exemplo da poda, do raleio e da colheita.

Macieira - A distribuição das espécies de tetraniquídeos nas macieiras foi oposta à encontrada sobre as plantas daninhas, de tal forma que $P$. ulmi representou $95 \%$ das ocorrências. O número de tetraniquídeos foi maior na PH (24-11 e 21-12-98) (Tabela 2), isto porque o deslocamento de tetraniquídeos no solo depende de sua cobertura vegetal (Zahner \& Baumgärtner, 1988). Assim, os tetraniquídeos que estavam sobre as plantas daninhas na entrelinhas, passam diretamente para as macieiras por não haver plantas daninhas para refúgio e alimentação na linha de plantio (Jusara-Bals, 1992). Na PSM, o deslocamento de tetraniquídeos do solo para as macieiras foi mais lento do que o verificado na $\mathbf{P H}$, possivelmente, porque os ácaros fitófagos possuíam alimento em abundância no solo. Observações de campo revelam que $N$. californicus migra para as macieiras em meados de dezembro (Monteiro, informação pessoal), provavelmente quando ocorre aumento da população de $P$. ulmi e do teor de cairomônios sobre as macieiras, como mostrou Collier et al. (2000). Quantidades significativas de ácaros predadores só foram observadas no levantamento de 6-1-99 (Tabela 2), nas parcelas em que não houve controle químico das plantas daninhas. $\mathrm{O}$ desenvolvimento de ácaros predadores foi lento e não representou obstáculos aos tetraniquídeos, principalmente na $\mathbf{P H}$, entre 21-12-98 a 6-1-99.

TABELA 2 - Flutuação de Neoseiulus californicus e tetraniquídeos sobre macieira, em três manejos de plantas daninhas, Agrícola Fraiburgo S.A., Fraiburgo, 1998.

\begin{tabular}{|c|c|c|c|c|c|c|}
\hline \multirow{2}{*}{ Data } & \multicolumn{3}{|c|}{ Neoseiulus californicus ${ }^{I}$} & \multicolumn{3}{|c|}{ Tetraniquídeo } \\
\hline & $\mathbf{P S M}^{2}$ & PR & PH & PSM & PR & PH \\
\hline 19-out-98 & 0 & 0 & 0 & $0,0 \mathrm{~B}$ & $1,0 \mathrm{~A}$ & $1,5 \mathrm{~A}$ \\
\hline $23-$ nov-98 & $0,1 \mathrm{~A}^{3}$ & $0,1 \mathrm{~A}$ & $0,0 \mathrm{~A}$ & $3,0 \mathrm{~B}$ & $23,4 \mathrm{~A}$ & $21,3 \mathrm{~A}$ \\
\hline 21-dez-98 & $0,1 \mathrm{~A}$ & $0,2 \mathrm{~A}$ & $0,1 \mathrm{~A}$ & $34,7 \mathrm{~B}$ & $23,0 \mathrm{C}$ & $80,4 \mathrm{~A}$ \\
\hline 6-jan-99 & $2,3 \mathrm{~A}$ & $2,5 \mathrm{~A}$ & $0,3 \mathrm{~B}$ & $25,1 \mathrm{~A}$ & $26,7 \mathrm{~A}$ & $19,4 \mathrm{~A}$ \\
\hline 20-jan-99 & $0,2 \mathrm{~A}$ & $0,2 \mathrm{~A}$ & $0,1 \mathrm{~A}$ & $1,0 \mathrm{~A}$ & $2,6 \mathrm{~A}$ & $0,5 \mathrm{~A}$ \\
\hline 18 -fev-99 & $1,5 \mathrm{~B}$ & $2,7 \mathrm{~A}$ & $1,1 \mathrm{C}$ & $4,3 \mathrm{~A}$ & 7,7A & $3,0 \mathrm{~A}$ \\
\hline 14-mai-99 & 0,0 & 0,0 & 0,0 & 0,0 & 0,0 & 0,0 \\
\hline
\end{tabular}

${ }^{1}$ Número médio de ácaros por folha de macieira

${ }^{2} \mathbf{P S M}$ - parcela sem manejo; PR - parcela roçada; $\mathbf{P H}$ - parcela herbicida.

${ }^{3}$ Médias seguidas pelas mesmas letras na linha e para cada tipo de ácaros não diferem estatisticamente, pelo teste de Duncan, a 5\% de probabilidade

O nível de controle de tetraniquídeos nas macieiras foi alcançado na PH em 21-12-98, o que levou à realização de uma pulverização em todas as parcelas (4-1-99) com o acaricida propargite. A baixa redução de ácaros fitófagos no dia 6-01-99 pode ser explicada pela lenta atividade de 
vapor de propargite sobre os tetraniquídeos, pois o pico de mortalidade é verificada após 4 a 5 dias da aplicação (Lachadenede et al., 1998). Observou-se, no levantamento de 20-1-99, que o número de ácaros predadores diminuiu em todas as parcelas, em função da redução da população de tetraniquídeos e não dos efeitos do acaricida, pois Monteiro $(1993 ; 1994)$ não verificou efeitos negativos deste acaricida sobre $N$. californicus. Mesmo com a redução do ácaro predador, todas as parcelas não apresentaram reinfestações significativas de tetraniquídeos.

\section{CONCLUSÃO}

Neoseiulus californicus foi mais abundante nas parcelas, cujos manejos proporcionaram desenvolvimento de plantas daninhas na linha de plantio, ocorrendo, igualmente, maiores quantidades de tetraniquídeos. $\mathrm{O}$ ácaro predador teve preferência em permanecer sobre Plantago tormentosa a Erigeron sp. A população de tetraniquídeos foi significativamente maior sobre as macieiras cultivadas nas parcelas cujas plantas daninhas foram controladas por herbicidas.

\section{AGRADECIMENTOS}

À Agrícola Fraiburgo S. A. pelo apoio a este trabalho.

\section{REFERÊNCIASBIBLIOGRÁFICAS}

ALTIERI, M. A; LIEBMAN, M. Insect, wed, and plant disease management in multiple cropping system. In. C. A. Francis (Ed.), Multiple cropping systems. New York, MacMillan Publishing, 1986.

BAILLOD, M.; VENTURI, I. Lutte biologique contre l'acarien rouge en viticulture. I. Répartition, distribution et méthode de contrôle des populations en prédateurs typhlodromes. Revue suisse Viticulture Arboriculture et Horticulture, Lausanne, 12: 231-238, 1980.

CARVALHO, J.F.C.L. Algumas considerações sobre um ácaro fitófago da macieira Panonychus ulmi Koch. Revista Agronômica, Lisboa Silva, v. 54, p.1-12, 1971

CASTAGNOLI, M.; AMATO, F. Studi di laboratório sull'interazione tra il predador Amblyseius californicus (McGregor) (Acari:Phytoseiidae) e la sua preda Tetranychus urticae Koch (Acarina: Tetranychidae). Redia, Florença, v. 74, n. 1, p. 77-85, 1991.

CASTAGNOLI, M.; SIMONI. S.; PINTUCCI, M. Response of a laboratory strain of Amblyseius californicus (McGregor) (Acari: Phytoseiidae) to semi-natural outdoor conditions. Redia, Florença, v. 78, n. 2, p. 273-282, 1995.

COSTA-COMELLES, J.; GARCIA-MARI,F;; FERRAGUT, F.; LABORDA, R.; MARZAL, C. Bioecologia de ácaros depredadores y fitófagos em manzanos de Lerida. In: CONGRESSO S.E.C.H., 2., 1986. Actas... p. 107-1016.

COLLIER, K. F. S. Interações tritróficas entre o ácaro predador Neoseiulus californicus (McGregor) (Acari: Phytoseiidae), ácaros fitófagos e suas plantas hospedeiras. 1998. 69f., Dissertação (Mestrado em produção vegetal), Universidade Estadual do Norte Fluminense, Campos de Goytacazes, 1998

COLLIER, K. F. S.; EIRAS, A. E.; ALBUQUERQUE, G. S.; BLACKMER, J. L. ARAÚJO, M. C.; MONTEIRO L. B. Localização de presas a curta distância por Neoseiulus californicus (McGregor) (Acari: Phytoseiidae): O papel dos aleloquímicos dos ácaros fitófagos Panonychus ulmi (Koch) e Tetranychus urticae Koch (Acari: Tetranychus) e da planta hospedeira, Malus domestica (Borkham). Anais Sociedade Entomológica do Brasil, Piracicaba, v. 29, n. 4, p. 705-713, 2000

FAUVEL, G.; BOURGOIN, B.; PERRON, G.; ROUZET, J. Importance de la colonisation des vergers de pommier et pecher du sud de la France par Neoseiulus californicus (McGregor) et consequences pour la lutte biologique contre l'araignee rouge Panonichus ulmi (Koch) (Acari: Phytoseiidae, Tetranychidae). In: CONFÉRENCE INTERNATIONALESUR RAVAGEURSENAGRICULTURE, 1993, Montpellier. p. 587-596.

LACHADENEDE J., F.; RAUCH; JACKSON, D. Propargite en arboriculture fruitière et en vigne. La Défense des Végétaux, Paris, 249-250, p. 33-37, 1998.

JUVARA-BALS, I. Ecossystème veger de pommier: analyse de l'interaction Gamasida - Tetranychidus urticae Koch (Acariens) au niveau du sol et de as coverture végétale, 1992. $110 \mathrm{f}$., Tese Doutorado Ciências biológicas, École Polytechnique Fédérale de Zurich, Zurich, 1992.

MONTEIRO, L. Controle biologique de Panonychus ulmi (Koch) (Acari: Tetranychidae) en fonction du programme de traitement contre la mouche des fruits dans la region de Vacaria. In: Conférence Internationale sur Ravageurs en Agriculture, Montpellier, 1993, p. 611-619.

MONTEIRO, L. Manejo integrado de Panonychus ulmi em macieira, primeiras experiências com a introdução de Neoseiulus californicus. Revista Brasileira de Fruticultura, Cruz das Almas, 16, p. 46-53, 1994.

PURVIS, G.; CURRY, J. P. The influence of sward management on foliage arthropod communities in a ley grassland. Journal of Applied Ecology, Oxford, 18, p. 711-725, 1981.

VAN DE VRIE, M. Control of Tetranychidae in crops. Greenhouse Ornamentals. In: HELLE, W.; SABELIS, M. (Ed.)., Spider mites, their biology, natural enemies and control. Amsterdan: 1985. v. 1b, p. $273-$ 282.

VET, L.E.M.; DICKE, M. Ecology of infochemichal use by natural enemies in a tritrophic context. Annual Review Entomology, College Park, 37, p. 141-172, 1992.

Zahner, Ph.; Baumgärtner, J. Analyse des insteractions plantetétranychus-phytoseiides. I. Modèles de populations pour la dynamique de Panonychus ulmi et Tetranychus urticae en vergers de pommiers. Acta Oecologica Oecologia Applicata, Montrouge, v. 9, p. 311-333, 1988 